\title{
PESO VIVO Y CRECIMIENTO DE CORDEROS MERINO PRECOZ, SUFFOLK Y MESTIZOS EN PRADERAS MEDITERRÁNEAS SEMIÁRIDAS DE CHILE
}

\section{LIVEWEIGHT AND GROWTH OF MERINO PRECOZ, SUFFOLK AND CROSSBRED LAMBS IN A SEMIARID MEDITERRANEAN GRASSLAND OF CHILE.}

\author{
Giorgio Castellaro G. ${ }^{1 *}$, Ximena García F. ${ }^{1}$, Juan Carlos Magofke S. ${ }^{1}$, y Gerónimo Marín F. ${ }^{1}$ \\ ${ }^{1}$ Universidad de Chile, Facultad de Ciencias Agronómicas, Dpto. Producción Animal, Santa Rosa \\ 11315, La Pintana, Santiago, Chile. \\ * Autor para correspondencia: gicastel@uchile.cl
}

\section{RESUMEN}

Con el objeto de evaluar el uso de cruzamiento terminal, se comparó el peso vivo y crecimiento entre el nacimiento y destete, de corderos Suffolk (S), Merino Precoz (MP) y mestizos Suffolk x Merino Precoz (SMP), pastoreando praderas mediterráneas de la zona semiárida de Chile. El ensayo comenzó con el encaste de las ovejas, finalizando con el destete, cuatro meses de iniciadas las pariciones. Se analizó el peso al nacer, peso ajustado a los 60; 90 y 120 días, y las ganancias de peso entre periodos. Previo al análisis estadístico los datos fueron corregidos por tipo de parto y edad de la madre. Las fuentes de variación fueron genotipo y sexo de los corderos. Estas variables fueron importantes, no obstante se observaron cambios en el orden de mérito de los genotipos evaluados según los periodos analizados. A los 90 días, $\mathrm{S}$ y SMP mostraron pesos vivos similares, con una ventaja importante de $\mathrm{S}$ respecto a MP (5,8\%). Sin embargo, a los 120 días, SMP registró pesos 5,4 y 11,6\% superior a $S$ y MP, respectivamente $(P \leq 0,05)$. Esto ocurrió porque entre los 90 y 120 días el incremento de peso diario fue 51,0 y $115,5 \%$ mayor que $S$ y MP, respectivamente. A pesar de que los machos pesaron más al nacer $(3,9 \%)$ y a los 90 días $(7,1 \%)$, a los 120 días esta diferencia no fue importante $(2,8 \% ; P=0,1637)$. Los resultados sugieren que el cruzamiento terminal se justificaría si el objetivo es obtener corderos sobre $35 \mathrm{~kg}$ al destete.

Palabras clave: cruzamiento terminal, ganancia de peso, razas ovinas.

\section{ABSTRACT}

In order to evaluate the use of terminal crossing, liveweight and growth of lambs from birth to weaning were compared in Suffolk (S), Merino (MP) and crossbred Suffolk x Merino (SMP), grazing on Chilean semiarid Mediterranean grasslands. The trial began with mating and finished with weaning, four months after lambing started. Variables analyzed were birth weight, adjusted liveweight at 60 , 90 and 120 days of age, and liveweight gain between these periods. Prior to statistical analysis, data were corrected by birth type and maternal age. Sources of variation were lambs' genotype and sex. Effects were important but significant changes were observed in the order of merit of the genotypes evaluated at different periods until weaning. At 90 days, liveweight of $\mathbf{S}$ and SMP was similar and only $S$ was significantly higher than MP (5.8\%). However, weight of SMP lambs was 5.4 and $11.6 \%$ higher than $S$ and MP at 120 days, respectively $(P \leq 0.05)$. This occurred because the daily liveweight gain between 90 and 120 days was $\mathbf{5 1 . 0}$ and $\mathbf{1 1 5 . 5 \%}$ higher than $\mathrm{S}$ and MP, respectively. Even though

Recibido: 25 mayo 2015. $\quad$ Aceptado: 4 julio 2016. 
males weighed more at birth $(3.9 \%)$ and at 90 days $(7.1 \%)$, this difference was not significant at 120 days $(2.8 \% ; \mathrm{P}=\mathbf{0 . 1 6 3 7})$. These results suggest that the terminal crossing used is justifiable only if the objective is to obtain lambs weighing 35 or more $\mathrm{kg}$ at weaning.

Key words: breeds of sheep, live-weight gain, terminal crossbreeding.

\section{INTRODUCCIÓN}

En secanos de la zona semiárida de Chile la producción ovina se ha desarrollado en condiciones extensivas. Se ocupan terrenos marginales con bajos niveles productivos de los pastizales, lo que permite una reducida carga por unidad de superficie, lográndose, en consecuencia, índices reproductivos y productivos que no superan el $85 \%$ de corderos al destete y $26 \mathrm{~kg}$ de peso vivo $\mathrm{ha}^{-1}$ (Crempien, 1996). Lo señalado se traduce, generalmente, en una baja rentabilidad (Castellaro, 2006). Según ODEPA (2013), la producción de carne ovina es un rubro que tiene posibilidades promisorias especialmente como producto de exportación. Para mejorar la situación actual es necesario, entre otras medidas, incrementar la eficiencia económica de la empresa mejorando aspectos biológicos, las condiciones de comercialización y de consumo por parte de la población.

El uso de cruzamientos terminales, unido a una adecuada época de servicio, permitiría incrementar la producción y el crecimiento de los corderos (Vinent et al., 2004; Bianchi, 2006). Resultados obtenidos por este último autor, indican que independiente de la raza materna, la utilización de una raza paterna carnicera en sistemas de cruzamientos terminales mejora significativamente el peso y la condición corporal de los corderos. Los autores afirman que mediante la incorporación de cruzamientos industriales es posible aumentar las ganancias de peso pre-destete y el peso de los corderos entre un 9 y un $17 \%$, respectivamente, aspectos que estarían condicionados por la raza paterna que interviene y la condición nutricional de la oveja.

García (1986), Sales et al. (2005) y Thomas (2008), coinciden en que la raza Suffolk Down tiene una alta tasa de crecimiento y una buena calidad de la canal, lo que la convierte en un opción apropiada para la producción de corderos a través de cruzamientos terminales. Según Daza (2002), este tipo de cruzamientos produciría mayores pesos de los corderos al nacer, lo que se traduciría en una mayor vitalidad y menor mortalidad de las crías durante el período de lactación, respecto de las razas puras. Esta técnica mejoraría además, las tasas de crecimiento diario entre 10 a $20 \%$, y la calidad de canal.

En los rebaños ovinos de la zona mediterránea central de Chile es común la crianza de razas de doble propósito, como Merino Precoz, la que dada su rusticidad logra una buena adaptación al medio, generalmente produce corderos con menores tasas de crecimiento y un rendimiento inferior de carcasa en comparación con otros genotipos de tipo carnicero. Una posibilidad de mejoramiento de las características antes mencionadas, lo constituye el cruzamiento terminal con razas de carne como Suffolk.

El objetivo de este estudio fue comparar características de crecimiento y peso vivo de corderos Suffolk y Merino Precoz, respecto a un cruzamiento terminal de machos Suffolk sobre hembras Merino adultas, en pastizales sin suplementación, del secano mediterráneo semiárido de la Región Metropolitana de Chile.

\section{MATERIALES Y MÉTODOS}

El estudio se realizó en la Estación Experimental Rinconada de Maipú (33³1' S; 7050’ O; 470 m.s.n.m.), perteneciente a la Facultad de Ciencias Agronómicas de la Universidad de Chile, durante el año 2007. En dicha temporada se registraron $128,2 \mathrm{~mm}$ de precipitación, lo que permite calificar al año como seco, pero con una distribución normal (Olivares et al., 1998). El ensayo comenzó con el encaste de las ovejas, desde el 2 de enero al 5 marzo de 2007, utilizando un $4 \%$ de carneros y finalizó con el destete de los corderos, aproximadamente 4 meses después de iniciados los partos. Se aparearon 155 vientres Suffolk y 120 vientres Merino Precoz, con 11 carneros Suffolk y 288 hembras Merino Precoz con 12 machos de la misma raza. Durante el periodo de apareamiento, las hembras de cada grupo de encaste permanecieron separadas junto con los respectivos carneros en dos potreros de similares características. Posteriormente al encaste, todas las ovejas fueron manejadas en conjunto, donde pastorearon sobre pastizales naturales (890 ha), con una carga animal de 1,21 equivalentes ovino seco ha-1 (Court et al., 2010), sin recibir ningún tipo de suplementación durante todo el ciclo productivo.

De estos apareamientos nacieron vivos 105 corderos Suffolk, 200 Merino Precoz y 108 mestizos Suffolk x Merino Precoz. Al momento del parto se registró la fecha de nacimiento, el sexo, la edad de la madre, el tipo de parto y el peso de los corderos. Dado que solo se aparearon hembras adultas MP de 5 y hasta 8 años, con carneros Su- 
ffolk para obtener los mestizos terminales, fue necesario corregir previamente los registros de peso vivo e incrementos de peso de los corderos de las razas puras a edad adulta de sus madres mediante la aplicación de un factor multiplicativo (Bradford, 2002), y determinar su habilidad para crecer independiente de la prolificidad. La corrección por tipo de parto se efectúo mediante la aplicación de un factor de corrección aditivo calculado utilizando los datos propios dentro de cada genotipo. Posterior a la corrección anterior, los pesos vivos de los corderos fueron ajustados por edad de la madre, expresándolos a edad de madre adulta (cinco o más años), mediante la aplicación de un factor de corrección de tipo multiplicativo, calculado con la información registrada en las madres $\mathrm{S}$, debido a que fueron las únicas que produjeron corderos provenientes de ovejas de diferentes edades.

Finalmente, con los datos de peso vivos corregidos, se estimaron los pesos ajustados a los 60; 90 y 120 días, utilizando la siguiente ecuación:

$$
P C_{t}=\left(P_{a}-P_{\text {nac }}\right) t \div E_{d}+P_{\text {nac }}
$$

dónde $P C_{t}$ es el peso ajustado a 60; 90 o 120 días $(\mathrm{kg}) ; P_{a}$ es el peso al momento del control; $P_{n a c}$ es el peso al nacimiento; $E_{d}$ es la edad del cordero y $t$ es la edad de ajuste del peso a los 60; 90 o 120 días. Conocidos los pesos a los 60; 90 y 120 días, se determinaron las ganancias promedio de peso (GDP, g día ${ }^{-1}$ ) entre dichos periodos.

Se asumió un diseño completamente al azar con estructura factorial (Kaps y Lamberson, 2004), considerando como fuentes de variación el genotipo, el sexo de los corderos y la interacción, el cual fue resuelto a través de un procedimiento de mínimos cuadrados, de acuerdo al modelo general lineal:

$$
Y_{i j k}=\mu+G_{i}+S_{j}+(G \times S)_{i j}+\varepsilon_{i j k}
$$

dónde $\mathrm{Y}_{\mathrm{ijk}}$ representa a la variable respuesta (pesos al nacimiento; 60; 90 y 120 días; incrementos de peso entre las edades anteriores); $\mu$ es el promedio general; $\mathrm{G}_{\mathrm{i}}$ es el genotipo del cordero; $\mathrm{S}_{\mathrm{i}}$ es el sexo del cordero y $(G \times S)_{i j}$ la interacción entre dichos factores y $\varepsilon_{\mathrm{ijk}}$ es el error experimental. La separación de medias se efectuó mediante el test de Student-Newman-Keuls (SNK) con un 95\% de significancia utilizando el software estadístico SAS (2003)

\section{RESULTADOS Y DISCUSIÓN}

El genotipo de los corderos y el sexo fueron, por lo general fuentes de variación importantes, no obstante, se observaron cambios relevantes de los efectos principales en las distintas etapas, lo cual afectó el orden de mérito de los genotipos evaluados. La interacción sexo-genotipo, sin embargo, nunca fue importante $(\mathrm{P}>0,05)$ (Tablas 1 a Tabla 6).

\section{Peso al nacer}

El peso al nacer de los mestizos Suffolk $x$ Merino Precoz (SMP) fue similar (P > 0,05) a los obtenidos por sus contemporáneos de las razas puras Merino Precoz (MP) y Suffolk (S). Los pesos al nacimiento de corderos $S$ fueron $7,1 \%$ superiores a los MP $(\mathrm{P} \leq 0,05)$ (Tabla 1$)$. Un comportamiento similar en esta etapa informan García et al. (2006). Los machos presentaron 3,9\% mayores pesos al nacimiento que las hembras ( $\mathrm{P}$ $\leq 0,05)$ (Tabla 2).

Tabla 1. Peso al nacer y pesos corregidos a los 60, 90 y 120 días (promedio \pm desviación estándar) de corderos Suffolk (S), Merino Precoz (MP) y mestizos Suffolk x Merino Precoz (SMP).

Table 1. Birth liveweight and corrected liveweight (mean \pm standard deviation) to 60,90 and 120 days

\begin{tabular}{|c|c|c|c|c|c|}
\hline \multirow[t]{2}{*}{ Peso vivo } & \multicolumn{3}{|c|}{ Genotipos } & \multicolumn{2}{|c|}{ Desvío } \\
\hline & Suffolk & Mestizos & Merino & SMP/S & SMP/MP \\
\hline & \multicolumn{3}{|c|}{---------------- kg --------------- } & \multicolumn{2}{|c|}{ 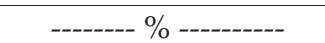 } \\
\hline Al nacimiento & $\begin{array}{c}4,85 \pm 0,69 \mathrm{a} \\
(\mathrm{n}=105)\end{array}$ & $\begin{array}{c}4,70 \pm 0,68 \mathrm{ab} \\
(\mathrm{n}=106)\end{array}$ & $\begin{array}{c}4,53 \pm 0,69 b \\
(\mathrm{n}=200)\end{array}$ & $-3,1$ & 3,8 \\
\hline A los 60 días & $\begin{array}{c}19,22 \pm 3,14 \mathrm{a} \\
(\mathrm{n}=91)\end{array}$ & $\begin{array}{c}19,13 \pm 3,06 \mathrm{a} \\
(\mathrm{n}=91)\end{array}$ & $\begin{array}{c}17,74 \pm 3,11 b \\
(n=169)\end{array}$ & $-0,5$ & 7,8 \\
\hline A los 90 días & $\begin{array}{c}28,12 \pm 4,00 \mathrm{a} \\
(\mathrm{n}=88)\end{array}$ & $\begin{array}{c}27,38 \pm 3,87 a b \\
(\mathrm{n}=91)\end{array}$ & $\begin{array}{c}26,59 \pm 3,94 b \\
(n=167)\end{array}$ & $-2,6$ & 3,0 \\
\hline A los 120 días & $\begin{array}{c}32,70 \pm 5,47 b \\
(n=90)\end{array}$ & $\begin{array}{c}34,45 \pm 5,34 \mathrm{a} \\
(\mathrm{n}=90)\end{array}$ & $\begin{array}{c}29,65 \pm 5,43 c \\
(n=164)\end{array}$ & 5,4 & 16,2 \\
\hline
\end{tabular}
of Suffolk (S), Merino Precoz (MP) and crossbred Suffolk x Merino Precoz (SMP) lambs.

Letras diferentes en el sentido horizontal indican diferencia significativas al test $\mathrm{SNK}(\mathrm{P} \leq 0,05)$.

SMP: corderos producto del apareamiento de carneros Suffolk sobre vientres Merino Precoz. 
Tabla 2. Peso al nacer y pesos corregidos a los 60, 90 y 120 días (promedio \pm desviación estándar) según el sexo, en corderos Suffolk, Merino Precoz y mestizos Suffolk x Merino Precoz.

Table 2. Birth liveweight and corrected liveweight to 60,90 and 120 days (mean \pm standard deviation) by sex, of Suffolk (S), Merino Precoz (MP) and crossbred Suffolk x Merino Precoz (SMP) lambs.

\begin{tabular}{|c|c|c|c|}
\hline \multirow[t]{2}{*}{ Peso vivo } & \multicolumn{2}{|c|}{ Sexo Cría } & \multirow{2}{*}{$\begin{array}{c}\text { Desvío } \\
\text { Machos/Hembras }\end{array}$} \\
\hline & Machos & Hembras & \\
\hline & \multicolumn{2}{|c|}{--------------- kg --------------- } & $\%$ \\
\hline $\mathrm{Al}$ nacimiento & $\begin{array}{c}4,78 \pm 0,71 \mathrm{a} \\
(\mathrm{n}=180)\end{array}$ & $\begin{array}{c}4,60 \pm 0,73 \mathrm{~b} \\
(\mathrm{n}=231)\end{array}$ & 3,9 \\
\hline A los 60 días & $\begin{array}{c}18,80 \pm 3,17 \mathrm{a} \\
(\mathrm{n}=152)\end{array}$ & $\begin{array}{c}18,59 \pm 3,25 a \\
(n=199)\end{array}$ & 1,1 \\
\hline A los 90 días & $\begin{array}{c}28,30 \pm 4,04 a \\
(\mathrm{n}=149)\end{array}$ & $\begin{array}{c}26,43 \pm 4,11 b \\
(\mathrm{n}=197)\end{array}$ & 7,1 \\
\hline A los 120 días & $\begin{array}{c}32,69 \pm 5,53 \mathrm{a} \\
(\mathrm{n}=149)\end{array}$ & $\begin{array}{c}31,85 \pm 5,65 \mathrm{a} \\
(\mathrm{n}=195)\end{array}$ & 2,6 \\
\hline
\end{tabular}

Letras diferentes en el sentido horizontal indican diferencia significativas al test $\mathrm{SNK}(\mathrm{P} \leq 0,05)$.

\section{Peso a los 60 días}

Como se observa en la Tabla 1 , el peso a los 60 días de los $\mathrm{S}$ y mestizos fue similar $(\mathrm{P}>0,05)$. Los corderos MP fueron significativamente más livianos a esta edad en comparación con los otros dos genotipos $(\mathrm{P} \leq 0,05)$. El $\mathrm{S}$ y SMP superaron al MP en un 7,1 y 3,8\%, respectivamente. Estos resultados muestran gran similitud con los informados por García et al. (2006). A esta edad machos y hembras (Tabla 2) tuvieron pesos similares $(\mathrm{P}>0,05)$.

\section{Peso a los 90 días}

A esta edad, nuevamente el genotipo S logró el mayor peso con un promedio de $28,1 \mathrm{~kg}$, manteniendo una diferencia significativa $(\mathrm{P} \leq 0,05)$ del $5,8 \%$ respecto a la raza MP. Los mestizos a esta edad, con un peso de 27,4 $\mathrm{kg}$, no se diferenciaron de sus contemporáneos $\mathrm{S}$ y MP $(\mathrm{P}>0,05)$ (Tabla 1). En este periodo los machos superaron a las hembras en un $7 \%(\mathrm{P} \leq 0,05)$ (Tabla 2$)$. Estos resultados son concordantes con lo mencionado por Leeds et al. (2012), quienes mencionan que el cruzamiento terminal sobre ovejas Rambouillet, utilizando machos Suffolk, en condiciones de pastoreo extensivo, mejora el crecimiento pre-destete de los corderos.

\section{Peso a los 120 días}

A esta edad se produjo un cambio importante en el orden de mérito de los genotipos, destacándose los corderos SMP con un peso promedio de $34,5 \mathrm{~kg}$., el cual fue 5,4 y 16,2\% mayor al de sus contemporáneos $\mathrm{S}$ y $\mathrm{MP}$, respectivamente $(\mathrm{P}$ $\leq 0,05$ ). Por primera vez los mestizos fueron el genotipo más destacado. El efecto del sexo del cordero a esta edad no fue importante $(\mathrm{P}>0,05)$ (Tabla 2). La literatura señala que en la medida que los corderos aumentan en edad y/o las condiciones nutricionales mejoran, la diferencia entre sexos se incrementa (Bianchi, 2006), no obstante, cuando la alimentación es restringida, las diferencias entre sexos no son apreciables (Bianchi y Garibotto, 2002; Bianchi et al., 2003). Esto explicaría los resultados antes expuestos, dado que como se mencionó anteriormente, el año fue seco, por lo tanto la oferta de MS de los pastizales probablemente fue menor. $\mathrm{Al}$ respecto, y considerando la pluviometría registrada en la temporada, se estima una producción anual de MS del orden de los $772 \mathrm{~kg} \mathrm{ha}^{-1}$, cifra que representa solo un $39,7 \%$, respecto a un año promedio (Le Houerou y Hoste, 1977).

\section{Incremento diario de peso entre el nacimiento y los 60 días}

La tasa de crecimiento diaria hasta los 60 días de los corderos $S$ y SMP fue similar, pero superior a la de los corderos MP en 8,6 y 7,7\%, respectivamente $(\mathrm{P}>0,05)$ (Tabla 3). Estudios realizados con corderos Suffolk en ambientes con mayor pluviometría por García et al. (2002) y Avendaño et al. (2003), muestran ganancias de hasta $314 \mathrm{~g} \mathrm{día}^{-1} \mathrm{en}$ este período, dejando en evidencia el elevado potencial de crecimiento del genotipo carnicero. El sexo de la cría en este intervalo de tiempo no fue importante $(\mathrm{P}>0,05)$.

\section{Incremento diario de peso entre los 60 y 90 días}

En este periodo, el incremento de peso de todos los genotipos fue similar ( $\mathrm{P}>0,05)$, y de mayor magnitud en relación a la observado entre el 
Tabla 3. Ganancia diaria de peso (promedio \pm desviación estándar) de los corderos Suffolk (S), Merino Precoz (MP) y mestizos Suffolk x Merino Precoz (SMP) en distintas etapas entre el nacimiento y destete a los 120 días.

Table 3. Liveweight gain (mean \pm standard deviation) of Suffolk (S), Merino Precoz (MP) and crossbred Suffolk x Merino Precoz (SMP) lambs in different stages between birth and weaning at 120 days.

\begin{tabular}{|c|c|c|c|c|c|}
\hline \multirow[t]{2}{*}{ Ganancia diaria de peso } & \multicolumn{3}{|c|}{ Genotipos } & \multicolumn{2}{|c|}{ Desvío } \\
\hline & Suffolk & Mestizos & Merino & SMP/S & SMP/MP \\
\hline & ----------- & - g día ${ }^{-1}$---- & ----------- & \multicolumn{2}{|c|}{-------" \% --------- } \\
\hline Nacimiento - 60 días & $\begin{array}{c}239 \pm 54 \mathrm{a} \\
(\mathrm{n}=91)\end{array}$ & $\begin{array}{c}237 \pm 52 \mathrm{a} \\
(\mathrm{n}=92)\end{array}$ & $\begin{array}{r}220 \pm 53 b \\
(\mathrm{n}=169)\end{array}$ & $-0,8$ & 7,7 \\
\hline 60 - 90 días & $\begin{array}{c}295 \pm 72 \mathrm{a} \\
(\mathrm{n}=88)\end{array}$ & $\begin{array}{c}280 \pm 70 a \\
(n=92)\end{array}$ & $\begin{array}{l}296 \pm 71 a \\
(n=167)\end{array}$ & $-5,1$ & $-5,4$ \\
\hline 90 - 120 días & $\begin{array}{c}157 \pm 130 \mathrm{~b} \\
(\mathrm{n}=86)\end{array}$ & $\begin{array}{c}237 \pm 126 \mathrm{a} \\
(\mathrm{n}=90)\end{array}$ & $\begin{array}{c}110 \pm 128 \mathrm{c} \\
(\mathrm{n}=162)\end{array}$ & 51,0 & 115,5 \\
\hline
\end{tabular}

Letras diferentes en el sentido horizontal indican diferencia significativas al test $\mathrm{SNK}(\mathrm{P} \leq 0,05)$.

SMP: corderos producto del apareamiento de carneros Suffolk sobre vientres Merino Precoz.

nacimiento y los primeros 60 días de vida. Destacan los corderos MP con una tasa de crecimiento $34,5 \%$ superior en relación al de la etapa anterior. Los corderos $\mathrm{S}$ y SMP también mejoraron, pero solo en un 23,4 y $18,1 \%$, respectivamente. Durante estos 30 días, el crecimiento de los corderos coincidió con los meses de agosto y septiembre, período en el cual las praderas anuales de clima mediterráneo presentan las mayores tasas de crecimiento acompañado con una alta calidad nutritiva (Castellaro y Squella, 2006; Court et al., 2010). La combinación de una alta disponibilidad unida a una elevada calidad del forraje a la que tuvieron acceso la unidad madre-cría contribuye a explicar los mayores incrementos diarios de peso observados en esta etapa en relación al periodo anterior. El efecto sexo fue importante $(\mathrm{P} \leq 0,05)$. Los machos tuvieron un incremento de peso diario $18,9 \%$ superior a las hembras. Lo observado confirma la relación que existe entre el efecto del sexo y el nivel nutricional señalado anteriormente.

Incremento diario de peso entre los 90 y 120 días

Entre los 90 y 120 días se obtuvieron los menores incrementos de peso en los tres genotipos evaluados, produciéndose nuevamente un cambio en el orden de mérito de los genotipos, los cuales observaron diferencias significativas $(\mathrm{P} \leq$ $0,05)$, siendo los corderos mestizos los que obtuvieron las mayores ganancias de peso. Estos corderos superaron en 51,0 y $115,5 \%$ a los corderos $S$ y MP, respectivamente (Tabla 3). Lo observado es diferente a lo informado para ganancia de peso de bovinos de carne, donde el efecto de la heterosis se exterioriza en una mayor magnitud, cuando no hay restricción nutricional (Magofke et al., 2005).
Estudios clásicos reportan porcentajes de heterosis del orden de 5,3\% para la ganancia de peso pre-destete en ovinos (Nitter, 1978), mientras que en bovinos de carne estas cifras varían entre 3,9 y $6,4 \%$ (Long, 1980). En esta etapa los mestizos tuvieron un incremento de peso muy similar al observado entre el nacimiento y los 60 días, y solo $15,4 \%$ menor respecto al obtenido entre los 60 y 90 días $(\mathrm{P}<0,05)$. En el mismo orden, el S y MP tuvieron una disminución de 34,3 y $46,8 \%$ y de 50,0 y $62,8 \%$ en relación a las etapas anteriores. El efecto sexo también mostró un importante cambio en esta etapa. Las hembras tuvieron un incremento de peso $23,4 \%$ mayor que los machos ( $\mathrm{P} \leq$ $0,05)$. La disminución en el incremento de peso respecto del observado entre los 60 y 90 días fue 53,8 y $30,1 \%$ en machos y hembras, respectivamente (Tabla 4).

\section{Incremento diario de peso entre el nacimiento y los 90 días}

La ganancia de peso entre el nacimiento y los 90 días del S y SMP fue similar con valores promedio de 259 y $252 \mathrm{~g}_{\text {día }}{ }^{-1}$, respectivamente. El MP presentó la tasa más baja con $244 \mathrm{~g}_{\text {día }}{ }^{-1}$. Esta diferencias solo fue significativa $(P<0,05)$ respecto al Suffolk (Tabla 5). Los machos crecieron a una tasa $8,7 \%$ superior respecto a las hembras ( $\mathrm{P}$ $\leq 0,05)$ (Tabla 6).

\section{Incremento diario de peso entre nacimiento-120 días}

Al considerar el periodo completo entre el nacimiento y el destete, que ocurrió a los 120 días, las tasas de crecimiento diario promedio de los genotipos evaluados fue significativamente dife- 
Tabla 4. Ganancia diaria de peso (promedio \pm desviación estándar) de los corderos machos y hembras, en distintos periodos entre el nacimiento y el destete.

Table 4. Liveweight gain (mean \pm standard deviation) of male and female lambs in different stages between birth and weaning at 120 days.

\begin{tabular}{|c|c|c|c|}
\hline \multirow[t]{2}{*}{ Ganancia diaria de peso } & \multicolumn{2}{|c|}{ Sexo Cría } & \multirow{2}{*}{$\begin{array}{c}\text { Desvío } \\
\text { Machos/Hembras }\end{array}$} \\
\hline & Machos & Hembras & \\
\hline & \multicolumn{2}{|c|}{ - } & $\%$ \\
\hline Nacimiento - 60 días & $\begin{array}{c}232 \pm 54 a \\
(n=153)\end{array}$ & $\begin{array}{c}232 \pm 56 \mathrm{a} \\
(\mathrm{n}=199)\end{array}$ & 0,0 \\
\hline 60 - 90 días & $\begin{array}{c}315 \pm 73 \mathrm{a} \\
(\mathrm{n}=149)\end{array}$ & $\begin{array}{r}265 \pm 74 b \\
(n=198)\end{array}$ & 18,9 \\
\hline 90 - 120 días & $\begin{array}{c}150 \pm 132 b \\
(\mathrm{n}=146)\end{array}$ & $\begin{array}{r}185 \pm 133 a \\
(n=192)\end{array}$ & $-18,9$ \\
\hline
\end{tabular}

Letras diferentes en el sentido horizontal indican diferencia significativas al test $\mathrm{SNK}(\mathrm{P} \leq 0,05)$.

Tabla 5. Ganancia diaria de peso (promedio desviación estándar) de los corderos Suffolk (S), Merino Precoz (MP) y mestizos Suffolk x Merino Precoz (SMP) entre el nacimiento y los 90 y el nacimiento y los 120 días.

Table 5. Liveweight gain (mean \pm standard deviation) of Suffolk (S), Merino Precoz (MP) and crossbred Suffolk x Merino Precoz (SMP) lambs between birth and 90 days, and birth and 120 days.

\begin{tabular}{|c|c|c|c|c|c|}
\hline \multirow[t]{2}{*}{ Ganancia diaria de peso } & \multicolumn{3}{|c|}{ Genotipos } & \multicolumn{2}{|c|}{ Desvío (\%) } \\
\hline & Suffolk (S) & Mestizo (SMP) & Merino (MP) & SMP/S & SMP/MP \\
\hline & \multicolumn{3}{|c|}{ - } & \multicolumn{2}{|c|}{ 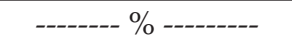 } \\
\hline Nacimiento - 90 días & $\begin{array}{c}259 \pm 45 a \\
(n=88)\end{array}$ & $\begin{array}{c}252 \pm 44 a b \\
(\mathrm{n}=91)\end{array}$ & $\begin{array}{c}244 \pm 44 b \\
(n=168)\end{array}$ & $-2,7$ & 3,3 \\
\hline Nacimiento - 120 días & $\begin{array}{c}232 \pm 40 b \\
(\mathrm{n}=90)\end{array}$ & $\begin{array}{c}250 \pm 39 a \\
(\mathrm{n}=89)\end{array}$ & $\begin{array}{c}211 \pm 40 \mathrm{c} \\
(\mathrm{n}=163)\end{array}$ & 7,8 & 18,5 \\
\hline
\end{tabular}

Letras diferentes en el sentido horizontal presentan diferencias significativas al test $\mathrm{SNK}(\mathrm{P} \leq 0,05)$.

SMP: corderos producto del apareamiento de carneros Suffolk sobre vientres Merino Precoz.

Tabla 6. Ganancia diaria de peso (promedio desviación estándar) de los corderos machos y hembras entre el nacimiento y los 90 y nacimiento y 120 días.

Table 6. Daily liveweight gain (mean \pm standard deviation) of male and female lambs between birth and 90 days, and birth and 120 days.

\begin{tabular}{|c|c|c|c|}
\hline \multirow[t]{2}{*}{ Variables } & \multicolumn{2}{|c|}{ Sexo Cría } & \multirow{2}{*}{$\begin{array}{c}\text { Desvío } \\
\text { Machos/Hembras }\end{array}$} \\
\hline & Machos & Hembras & \\
\hline & \multicolumn{2}{|c|}{-------------- g día-1 ----------- } & $\%$ \\
\hline Nacimiento - 90 días & $\begin{array}{c}262 \pm 45 a \\
(n=149)\end{array}$ & $\begin{array}{c}241 \pm 46 \mathrm{~b} \\
(\mathrm{n}=198)\end{array}$ & 8,7 \\
\hline Nacimiento - 120 días & $\begin{array}{c}235 \pm 40 a \\
(n=148)\end{array}$ & $\begin{array}{c}227 \pm 41 \mathrm{a} \\
(\mathrm{n}=194)\end{array}$ & 3,5 \\
\hline
\end{tabular}

Letras diferentes en el sentido horizontal presentaron diferencias significativas al test $\mathrm{SNK}(\mathrm{P} \leq 0,05)$. 
rentes $(\mathrm{P} \leq 0,05)$. Los corderos mestizos presentaron la mayor tasa de crecimiento promedio durante toda la lactancia, seguida por S y luego MP (Tabla 5). Al analizar el efecto sexo, sobre el crecimiento promedio de los corderos durante toda la lactancia, la diferencia no fue significativa $(\mathrm{P}>0,05)$.

\section{Comportamiento de los mestizos terminales}

En un cruzamiento terminal como el que se analiza, a pesar de que la heterosis materna no se manifiesta, se logra la exteriorización del $100 \%$ de la heterosis individual confundida con los efectos directo y materno de las razas involucradas. A pesar que no es posible estimar la heterosis individual debido a la ausencia del cruzamiento recíproco MPS (Magofke y García, 2002), en la Fig. 1 se presentan los pesos vivos y los incrementos de peso de los mestizos respecto al promedio de las razas puras, los cuales estiman en forma confundida los efectos directo y materno y el de la heterosis individual.

Hasta los 90 días el desvío del peso vivo de los corderos mestizos respecto al promedio de las razas puras fue pequeño, dado que no superó el 3,5\% (Fig. 1). Una situación similar ocurrió con los incrementos de peso, los cuales en algunos casos fueron incluso negativos. Solo entre los 90 y 120 días se observó una importante superioridad de los mestizos respecto al promedio de los corderos de las razas S y MP, principalmente en las ganancias de peso, donde se obtuvo un desvío promedio de $84,4 \%$ (236 versus $128 \mathrm{~g} \mathrm{día}^{-1}$, en corderos mestizos y puros, respectivamente) (Fig. 1). Llama la atención que en el último periodo, los corderos SMP no presentaran disminuciones en el incremento de peso tan altas como las observadas en los corderos contemporáneos de las razas puras $\mathrm{S}$ y $\mathrm{MP}$, a pesar de que en todo el periodo experimental, el manejo y la alimentación fueron iguales. El comportamiento registrado en este estudio también lo observaron García et al. (2002) en un ambiente con mayores precipitaciones $(500 \mathrm{~mm})$. En este estudio se evaluó durante dos temporadas, el crecimiento de corderos de la raza Suffolk con mestizos 50\% Suffolk, 25\% Finnish Landrace y 25\% Merino Precoz, a los que se les denominó Fisume. Los autores informan que el Suffolk pesó 16,8 y 5,4\% más que los mestizos al nacer y a los 60 días, respectivamente, sin embargo entre los 60 y 120 días el incremento de peso de los corderos Fisume únicos fue 10,2\% mayor, lo cual hizo que el peso fuera similar a los 120 días en este grupo de animales.

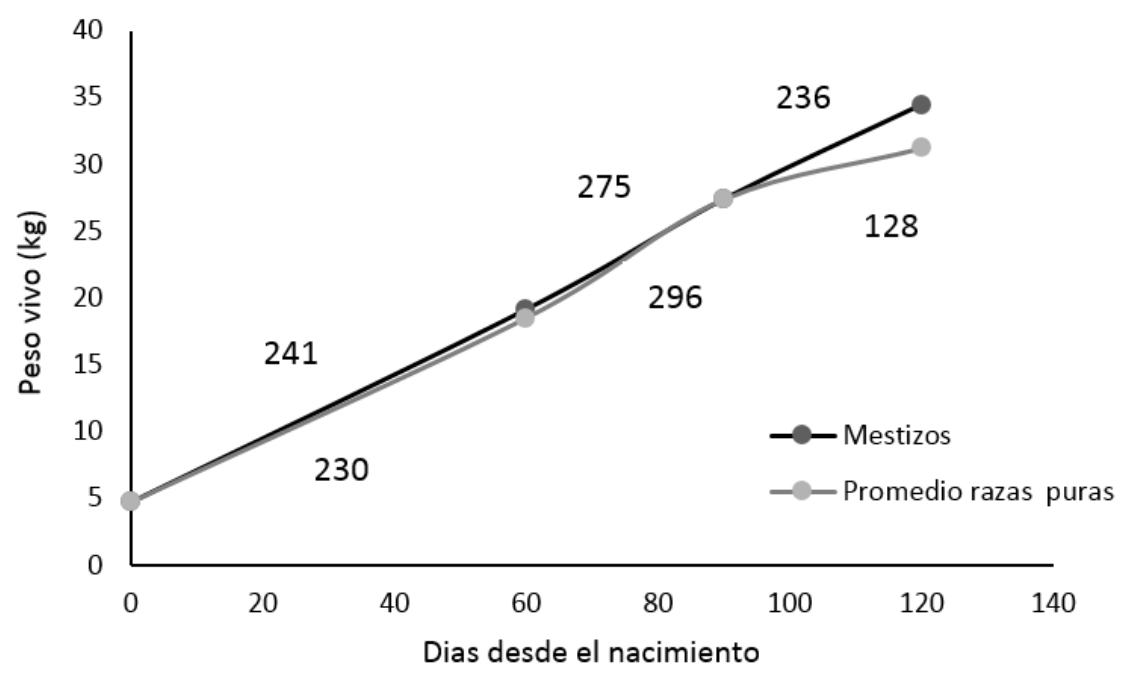

Fig. 1. Peso vivo de los corderos mestizos respecto al promedio de las razas puras Suffolk y Merino Precoz, en distintas etapas hasta el destete. Las cifras sobre y bajo las curva de peso vivo, indican la ganancias diaria de peso ( $\mathrm{g} \mathrm{día}^{-1}$ ) de los corderos mestizos y el promedio de los corderos puros, respectivamente.

Fig. 1. Liveweight of crossbred lambs compared to the average of pure breeds Suffolk and Merino Precoz, in various stages until weaning. Numbers above and below the curve of body weight indicate the daily weight gain $\left(\mathrm{g} \mathrm{day}^{-1}\right)$ of the crossbred lambs and the average of the pure lambs, respectively. 
Para explicar el comportamiento observado en este estudio durante el último período de la lactancia, es necesario considerar la posibilidad que intervinieran varias causales las que se detallan a continuación. A fines de septiembre en la pradera comienza un proceso de maduración, seguido de una acelerada senescencia, lo que va acompañado de una disminución en la calidad nutritiva (Castellaro y Squella, 2006; Court et al., 2010). Debido a esto, a inicios de octubre los corderos probablemente tuvieron disponible para consumir una pradera de menor calidad con una ingesta de leche muy reducida al final de este periodo (SCA, 2007). Como consecuencia de lo anterior, es lógico esperar una tasa de crecimiento menor a la registrada en las etapas anteriores. El mayor incremento promedio de peso observado en los corderos mestizos hacia el final de la lactancia, fue significativamente superior a corderos $S$ y MP $(P \leq 0,05)$, lo que podría atribuirse a que estos pudieron ingerir más leche que sus contemporáneos $\mathrm{S}$ y MP. Un motivo podría deberse a que por tener un mayor peso metabólico respecto a los MP puros habrían tenido una mayor capacidad para ingerir leche (Dove, 1988).

Por otra parte las madres de los corderos mestizos eran ovejas MP, las que en estudios realizados por Manterola et al. (2007) han demostrado poseer mayores niveles de producción de leche y persistencia de la lactancia respecto a las ovejas Suffolk. Por último, existen autores como Bianchi (2006) que señalan que los mayores beneficios de los cruzamientos terminales se obtienen a los 120 días o en corderos de 35 a $40 \mathrm{~kg}$. El autor agrega que, para lograrlo, el sistema de producción debe implementar un manejo que permita mantener la unidad madre-cría por más tiempo. Otros autores también sugieren que la ventaja de mantener por más tiempo la unidad madre-cría, es claramente observable cuando los pesos al sacrificio son superiores a los 32 kg (Furnival y Corbett, 1976; Purchas, 1978; Gaggero y Rodríguez, 1978; Bianchi y
Garibotto, 2002).

Una variable de gran importancia íntimamente relacionada con el incremento de peso, es el tiempo necesario para que los corderos alcancen un peso objetivo. El tiempo requerido para alcanzar $25 \mathrm{~kg}$ en el ensayo no presentó diferencias importantes entre los genotipos (Tabla 7). A los $30 \mathrm{~kg}$ se observaron diferencias hasta de 20 días a favor del mestizo respecto de la raza Merino Precoz. Con pesos objetivos superiores a $35 \mathrm{~kg}$ destaca el mestizaje, obteniéndose diferencias de 43 días en comparación con la raza Merino Precoz y de 12 días con respecto al Suffolk. Sobre la base de los resultados presentados en las condiciones en que se llevó a cabo este ensayo y la información de literatura revisada, hace posible comprender que los cambios en el nivel de mérito de los genotipos depende de la edad en que ocurra el destete, lo cual es consecuencia de la interacción de varios factores.

La información presentada pone en evidencia que el uso de cruzamientos terminales es una herramienta muy dependiente del manejo del rebaño y de sus objetivos. Finalmente, es importante destacar que la ventaja de una opción de cría con venta de corderos cercana al destete es función de los kilogramos de cordero destetados por oveja expuesta a carnero. La fertilidad y prolificidad de los vientres unido a la sobrevivencia de los corderos también son factores relevantes.

\section{CONCLUSIONES}

Bajo las condiciones en que se desarrolló este estudio, el comportamiento de los corderos $\mathrm{S}$ y SMP fue similar hasta los 90 días de edad. Los corderos MP generalmente presentaron menores pesos y velocidad de crecimiento. La superioridad de los corderos mestizos se expresa hacia fines del periodo de la lactancia (90-120 días), por lo cual el cruzamiento terminal, utilizando como raza materna Merino Precoz y como raza pater-

Tabla 7. Edad estimada para lograr diferentes pesos vivos objetivos en corderos Suffolk, Merino precoces y mestizos.

Table 7. Estimated age to achieve different target liveweights in Suffolk, Merino Precoz and crossbred lambs.

\begin{tabular}{cccc}
\hline Peso objetivo & \multicolumn{3}{c}{ Genotipos } \\
\hline & Merino & Mestizo & Suffolk \\
\hline $\mathrm{kg}$ & $-\mathrm{-}---$------------- días $^{------------------~}$ \\
20 & 68 & 64 & 63 \\
25 & 85 & 82 & 80 \\
30 & 121 & 101 & 103 \\
35 & 166 & 123 & 135 \\
\hline
\end{tabular}


na Suffolk, solo se justifica cuando el objetivo es producir corderos de mayor peso al destete con al menos $35 \mathrm{~kg}$ de peso vivo, lo que requiere mantener por más tiempo la unidad oveja-cordero.

\section{LITERATURA CITADA}

Avendaño, J., X. García, J.C. Magofke, y A. Galarza. 2003. Comportamiento de carneros Suffolk y mestizos 50\% Finnish-50\% Merino al ser apareados con ovejas Suffolk. Avances en Producción Animal 28:15-26.

Bianchi, G. 2006. Alternativas tecnológicas para la producción de carne ovina de calidad en sistemas pastoriles. 283 p. Editorial Hemisferio Sur, Montevideo, Uruguay.

Bianchi, G., y G. Garibotto. 2002. Influencia del sexo y del largo de lactancia sobre características de crecimiento, composición de la canal y calidad de carne de corderos. Montevideo, Uruguay. Produccion Ovina 15:71-92.

Bianchi, G., G. Garibotto, y O. Bentancur. 2003. Efecto del sexo y del largo de lactación sobre el desempeño de corderos Corriedale pesados. p. 7-9. En III Congreso Latinoamericano de Especialistas en Pequeños Rumiantes y Camélidos Sudamericanos. 7-9 de mayo 2003. Viña del Mar, Chile. Asociación Latinoamericana de Especialistas en Pequerños Rumiantes y Camélidos sudamericanos (ALEPRYCS), Santiago, Chile.

Bradford, E. 2002. Breeding and selection. p. 1-80. In Sheep Production Handbook. American Sheep Industry Association, Vol. 7. Fort Collins, Colorado. USA

Castellaro, G. 2006. Algunos elementos básicos para el desarrollo de sistemas de producción ovina en la zona sur del país. Departamento de Producción Animal, Universidad de Chile, Circular de Extensión 32:1-11.

Castellaro, G., y F. Squella. 2006. Modelo simple de simulación para la estimación del crecimiento, fenología y balance hídrico de praderas anuales de clima mediterráneo. Agricultura Técnica Chile 66(3):271-282.

Court, J., J. Webb-Ware, and S. Hides. 2010. Sheep Farming for Meat \& WooI. 322 p. CSIRO Publishing, Department of Primary Industries, Victoria, Australia.

Crempien, C. 1996. La pradera en los sistemas de producción ovina. p. 665-678. En Ruiz, I. (ed.). Praderas para Chile $2^{a}$ ed. Instituto de Investigaciones Agropecuarias, Santiago, Chile.

Daza, A. 2002. Mejora de la productividad y planificación de explotaciones ovinas. 232 p. Editorial Agrícola Española S.A, Madrid, España.
Dove, H. 1988. Estimation of the intake of milk by lambs, from the turnover of deuterium- or tritium-labelled water. British Journal of Nutrition 60:375-387.

Furnival, E.P., and J.L. Cobett. 1976. Early weaning of grazing sheep. I. Growth of lambs. Australian Journal of Experimental Agriculture and Animal Husbandry 16(79):149- 155.

Gaggero, C., y A. Rodríguez. 1978. Destete de corderos a temprana edad. Secretariado Uruguayo de la Lana (SUL), Boletín Técnico 3:1726.

García, G. 1986. Producción Ovina. 344 p. Facultad de Ciencias Agronómicas, Departamento de Producción Animal, Universidad de Chile, Santiago, Chile.

García, X., J. Avendaño, y J.C. Magofke. 2002. Comportamiento de la raza Suffolk y de Mestizo originados de Suffolk, Finnish Landrace y Merino, en dos épocas de encaste. I Peso y condición corporal de las ovejas y crecimientos de los corderos. Avances en Producción Animal 27:43-56.

García, X., J.C. Magofke, y C. Aubert. 2006. Comportamiento productivo del Merino Precoz y Suffolk Down en el secano interior de la Región Metropolitana, Chile. Avances en Producción Animal 32:35-56.

Kaps, M., and W. Lamberson. 2004. Biostatistics for Animal Science. 445 p. CABI Publishing, Oxfordshire, UK.

Leeds T.D., D.R. Notter, K.A. Leymaster, M.R. Mousel, and G.S. Lewis. 2012. Evaluation of Columbia, USMARC-Composite, Suffolk, and Texel rams as terminal sires in an extensive rangeland production system: I. Ewe productivity and crossbred lamb survival and preweaning growth. J. Anim. Sci. 90:2931-2940.

Le Houerou, H.N., and C.H. Hoste. 1977. Rangeland production and annual rainfall relations in the Mediterranean Basin and in the African Sahelo-Sudanian Zone. J. Range Manage. 30(3):181-189.

Long, C.R. 1980. Crossbreeding for beef production. J. Anim. Sci. 51:1197-1223.

Manterola, H., D. Cerda, y J. Mira. 2007. Producción y composición de la leche en ovejas Merino, Suffolk y Suffolk x Merino. p. 98-100. En Vo Congreso de Especialistas en Pequeños Rumiantes y Camélidos Sudamericanos. 2, 3 y 4 de mayo de 2007. Instituto Nacional de Tecnología Agropecuaria (INTA), Universidad Juan Agustín Maza, Universidad de Buenos Aires, Mendoza, Argentina. 
Magofke, J.C., y X. García. 2002. Uso de cruzamientos entre razas para mejorar la productividad animal. IV. Diseño y comparaciones requeridas para estimar el mérito de las razas puras y el vigor híbrido individual y materno. Departamento de Producción Animal, Universidad de Chile. Circular de Extensión 28:62-71.

Magofke, J.C., X. García, y H. González. 2005. Razas bovinas de carne y nuevos criterios de selección. 199 p. Publicación Docente $\mathrm{N}^{\circ} 18$. Departamento de Producción Animal, Facultad de Ciencias Agronómicas, Universidad de Chile. Santiago, Chile.

Nitter, G. 1978. Breed utilization for meat production in sheep. Animal Breeding Abstract. 46:131-143.

ODEPA. 2013. Estudio: Mercado de la carne y lana ovina en Chile. Informe Final. 331 p. Facultad de Agronomía - Universidad de Concepción. Ministerio de Agricultura, Oficina de Estudios y Políticas Agrarias (ODEPA), Santiago, Chile. .

Olivares, A., M. Johnston, y X. Contreras.1998. Régimen pluviométrico del secano interior de la Región Metropolitana. Avances en Producción Animal 23:35-43.
Purchas, R.W. 1978. A comparision of the fatness of weaned and unweaned lambs. New Zealand Journal Agriculture Research 21:211216.

Sales, F, E. Latorre, y R. Lira. 2005. Razas ovinas, p. 17-38. In Mujica. F. (ed.) Razas ovinas y caprinas en el Instituto de Investigaciones Agropecuaria (INIA). Boletin INIA $\mathrm{N}^{\circ} 127$, INIA, Osorno, Chile.

SAS. 2003. The SAS system for Windows. Release 9.1.3. SAS Institute, Cary, North Carolina, USA.

SCA. 2007. Nutrient requirement of domesticated ruminants. 296 p. Standing Committee on Agriculture (SCA), Ruminants Subcommittee. CSIRO Publishing, Victoria, Australia.

Thomas, D.L. 2008. Differences among breeds of sheep in the U.S. and their use in efficient sheep production systems. Department of Animal Sciences; University of Wisconsin, Madison, Wisconsin. Available at http:// www.uwex.edu/ces/animalscience/sheep/ wisline_03/thomas_handout.doc (Accessed 15 March 2008).

Vinent, J., D. Miñon, M. Alvarez, H. Giorgetti, G. Rodriguez, y A. Perlo. 2004. Cruzamientos industriales para producción de carne ovina. Boletín INTA 7:159-169. 